\title{
Conjugate Additions of Sulfur-stabilized Anions to Unsaturated Lactams. Synthesis of
}

\section{Polyfunctionalized benzo[ $a]$ quinolizinone systems}

Eva García, Esther Lete, * and Nuria Sotomayor

Departamento de Química Orgánica II, Facultad de Ciencia y Tecnología, Universidad del País Vasco / Euskal Herriko Unibertsitatea. Apdo. 644.48080 Bilbao (Spain) esther.lete@ehu.es

\section{Supporting Information}

Contents of file 1 (current file)

- General experimental methods

- Synthesis of $N$-[2-(3,4-dimethoxyphenyl)ethyl]glutarimide (1)

- Synthesis of 9,10-dimethoxy-1,2,3,6,7,11b-hexahydrobenzo[a]quinolizin-4-one (2a).

Procedure A

Procedure B

- Synthesis of 9,10-dimethoxy-11b-methyl-1,2,3,6,7,11b-hexahydrobenzo[a]quinolizin-4-one (2b).

- Synthesis of 9,10-dimethoxy-1,6,7,11b-tetrahydrobenzo[a]quinolizin-4-one (3a)

- Synthesis of 9,10-dimethoxy-11b-methyl-1,6,7,11b-tetrahydrobenzo[a]quinolizin-4-one $(3 \mathbf{b})$

- References

\section{Contents of file 2}

- Copies of ${ }^{1} \mathrm{H}$ NMR and ${ }^{13} \mathrm{C}$ NMR of compounds 
General experimental methods: Melting points were determined in unsealed capillary tubes and are uncorrected. IR spectra were obtained on $\mathrm{KBr}$ pellets (solids) or $\mathrm{CHCl}_{3}$ solution (oils). NMR spectra were recorded at $20-25{ }^{\circ} \mathrm{C}$, running at $250 \mathrm{MHz}$ or $500 \mathrm{MHz}$ for ${ }^{1} \mathrm{H}$ and $62.8 \mathrm{MHz}$ or $125.7 \mathrm{MHz}$ for ${ }^{13} \mathrm{C}$ in $\mathrm{CDCl}_{3}$ solutions. Assignment of individual ${ }^{13} \mathrm{C}$ resonances is supported by DEPT and HMQC experiments. Mass spectra were recorded under electron impact at $70 \mathrm{eV}$. GC-MS analyses were performed using a methyl polysiloxane column $(30 \mathrm{~m} \times 0.25 \mathrm{~mm} \times 0.25 \mu \mathrm{m})$. HRMS analyses were performed under electron impact with a TOF analyzer. TLC was carried out with $0.2 \mathrm{~mm}$ thick silica gel plates. Visualization was accomplished by UV light. Flash column chromatography ${ }^{1}$ on silica gel was performed with Kiesegel 60 (230-400 mesh). HPLC All solvents used in reactions were anhydrous and purified according to standard procedures. ${ }^{2}$ Organolithium reagents were titrated with diphenylacetic acid periodically prior to use. All air- or moisture-sensitive reactions were performed under argon; the glassware was dried $\left(130^{\circ} \mathrm{C}\right)$ and purged with argon.

\section{Synthesis of $N$-[2-(3,4-dimethoxyphenyl)ethyl]glutarimide (1).}<smiles>COc1ccc(CCN2C(=O)CCCC2=O)cc1OC</smiles>

1

A solution of glutaric anhydride (3 g, $26.3 \mathrm{mmol})$ and 2-(3,4dimethoxyphenyl)ethylamine $(1.0 \mathrm{~g}, 14.5 \mathrm{mmol})$ in glacial $\mathrm{AcOH}(30 \mathrm{~mL})$ was heated under reflux for $16 \mathrm{~h}$. The solvent was evaporated under vacuum and the resulting crude mixture was crystallized form $\mathrm{MeOH}$ to afford glutarimide 1 as a white solid (3 g, 75\%): $\mathrm{mp}(\mathrm{MeOH}) 111-112^{\circ} \mathrm{C}\left(\mathrm{lit}^{3} \mathrm{mp}(\mathrm{MeOH}) 111-113{ }^{\circ} \mathrm{C}\right)$; IR (KBr) 1725, $1690 \mathrm{~cm}^{-1} ;{ }^{1} \mathrm{H}$ NMR $\left(\mathrm{CDCl}_{3}\right)$ 1.83-1.94 (m, 2H), $2.61(\mathrm{t}, J=6.5 \mathrm{~Hz}, 4 \mathrm{H}), 2.71-2.77(\mathrm{~m}$, 2H), $3.85(\mathrm{~s}, 3 \mathrm{H}), 3.87(\mathrm{~s}, 3 \mathrm{H}), 3.93-3.99(\mathrm{~m}, 2 \mathrm{H}), 6.77$ (broad s, 3H); ${ }^{13} \mathrm{C} \mathrm{NMR}\left(\mathrm{CDCl}_{3}\right)$ 17.0, 32.7, $33.5,40.7,55.7,110.9,111.9,120.8,130.9,147.3,148.5,172.3$; MS (EI) $m / z$ (rel intensity) $277\left(\mathrm{M}^{+}, 8\right)$, 164 (100), 151 (25), 121 (4), 107 (6), 91 (6), 77, (4), 55 (20), 42 (8).Anal. Calcd for $\mathrm{C}_{15} \mathrm{H}_{19} \mathrm{NO}_{4}$ : C, 64.97; H, 6.91; N, 5.05. Found: C, 64.88; H, 6.85; N, 5.12. 


\section{Synthesis of 9,10-dimethoxy-1,2,3,6,7,11b-hexahydrobenzo[a]quinolizin-4-one (2a).}

\section{Procedure A.}<smiles>COc1cc2c(cc1OC)[C@H]1CCCC(=O)N1CC2</smiles>

$2 a$

$\mathrm{NaBH}_{4}(210 \mathrm{mg}, 5.6 \mathrm{mmol})$ was added in portions to a solution of glutarimide $1(393 \mathrm{mg}, 1.4 \mathrm{mmol})$ in $10 \% \mathrm{EtOH} / \mathrm{H}_{2} \mathrm{O}(20 \mathrm{~mL})$ at $0{ }^{\circ} \mathrm{C}$, maintaining $\mathrm{pH}=8$ by portion wise addition of $3 \mathrm{M} \mathrm{HCl}$. The reaction mixture was stirred for $40 \mathrm{~min} .3 \mathrm{M} \mathrm{HCl}$ was added to adjust $\mathrm{pH}$ to 4 , and water was added $(10 \mathrm{~mL})$. The mixture was extracted with $\mathrm{CH}_{2} \mathrm{Cl}_{2}(3 \times 20 \mathrm{~mL})$. The combined organic extracts were washed with saturated $\mathrm{NaHCO}_{3}(3 \times 10 \mathrm{~mL})$, and with brine $(3 \times 10 \mathrm{~mL})$, dried $\left(\mathrm{Na}_{2} \mathrm{SO}_{4}\right)$, and concentrated in vacuo. The tautomeric mixture of hydroxylactam and oxoamide was submitted to cyclization without further purification. Thus, TFA $(1.5 \mathrm{~mL}, 9 \mathrm{mmol})$ was added over a solution of the crude reaction mixture in $\mathrm{CH}_{2} \mathrm{Cl}_{2}(30 \mathrm{~mL})$, and the mixture was stirred at $\mathrm{rt}$ for $16 \mathrm{~h}$. The reaction was quenched by addition of saturated $\mathrm{NaHCO}_{3}(10 \mathrm{~mL})$. The organic phase was separated, and the resulting aqueous phase was extracted with $\mathrm{CH}_{2} \mathrm{Cl}_{2}(3 \times 10 \mathrm{~mL})$. The combined organic extracts were washed with brine $(3 \times 10 \mathrm{~mL})$, dried $\left(\mathrm{Na}_{2} \mathrm{SO}_{4}\right)$, and concentrated in vacuo. Flash column chromatography (silica gel, AcOEt) afforded benzoquinolizidone 2a (302 g, 83\%): mp (50\% hexane/AcOEt) $114-115^{\circ} \mathrm{C}$ (lit. ${ }^{4} \mathrm{mp}\left(\mathrm{EtO}_{2}\right)$ 88-90 $\left.{ }^{\circ} \mathrm{C}\right)$; IR (KBr) $1635 \mathrm{~cm}^{-1} ;{ }^{1} \mathrm{H}$ NMR $\left(\mathrm{CDCl}_{3}\right)$ 1.56-1.71 (m, 1H), 1.73-2.01 (m, 2H), 2.27-2.48 (m, 1H), 2.50-2.64 (m, 3H), 2.71-2.94 (m, 2H), $3.84(\mathrm{~s}, 6 \mathrm{H}), 4.58(\mathrm{dd}, J=10.3,4.4 \mathrm{~Hz}$, 1H), 4.81-4.88 (m, 1H), $6.59(\mathrm{~s}, 1 \mathrm{H}), 6.65(\mathrm{~s}, 1 \mathrm{H}) ;{ }^{13} \mathrm{C} \mathrm{NMR}\left(\mathrm{CDCl}_{3}\right)$ 19.2, 28.2, 30.6, 31.8, 39.6, 55.7, 55.9, 56.5, 107.9, 111.2, 127.0, 128.7, 147.4, 147.5, 169.6; MS (EI) $m / z$ (rel intensity) $262\left(\mathrm{M}^{+}+1,21\right)$ $261\left(\mathrm{M}^{+}, 100\right), 260$ (47), 246 (30), 230 (34), 205 (7), 191 (72), 176 (20), 146 (4), 91 (5). Anal. Calcd for $\mathrm{C}_{15} \mathrm{H}_{19} \mathrm{NO}_{3}: \mathrm{C}, 68.94 ; \mathrm{H}, 7.33 ; \mathrm{N}, 5.36$. Found: $\mathrm{C}, 68.98 ; \mathrm{H}, 7.55 ; \mathrm{N}, 4.91$. 


\section{Procedure B.}

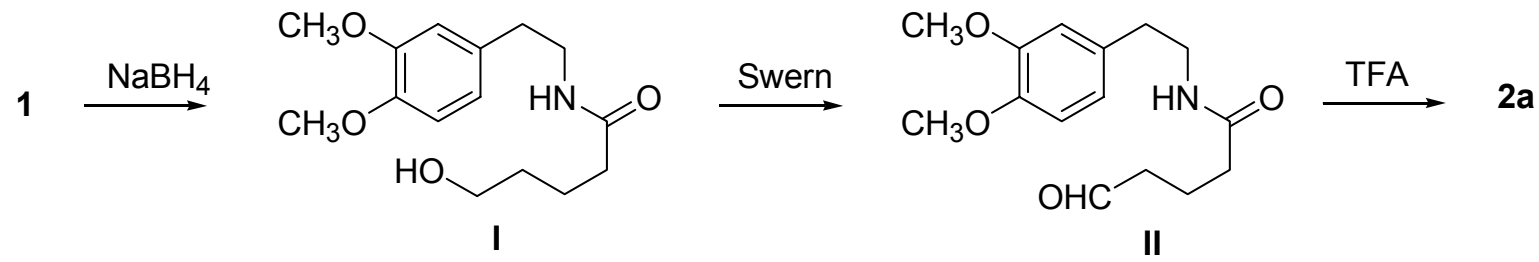

Treatment of glutarimide 1 ( $3 \mathrm{~g}, 11 \mathrm{mmol})$ with $\mathrm{NaBH}_{4}(2.87 \mathrm{~g}, 70 \mathrm{mmol})$ according to the procedure described in Procedure A gave the $N$-[2-(3,4-dimethoxyphenyl)ethyl]-5-hydroxypentanamide (I) (2.5 g, 82\%): $\mathrm{mp}\left(\mathrm{Et}_{2} \mathrm{O}\right) 71-72{ }^{\circ} \mathrm{C}$; IR ( $\left.\mathrm{KBr}\right) 3500,1637 \mathrm{~cm}^{-1} ;{ }^{1} \mathrm{H}$ NMR $\left(\mathrm{CDCl}_{3}\right)$ 1.46-1.57 (m, 2H), 1.61-1.73 $(\mathrm{m}, 2 \mathrm{H}), 2.15(\mathrm{t}, J=7.1 \mathrm{~Hz}, 2 \mathrm{H}), 2.66(\operatorname{broad~s}, 1 \mathrm{H}), 2.72(\mathrm{t}, J=7.1 \mathrm{~Hz}, 2 \mathrm{H}), 3.45(\mathrm{dd}, J=7.1,6.7 \mathrm{~Hz}$ $2 \mathrm{H}), 3.57(\mathrm{t}, J=5.9 \mathrm{~Hz}, 2 \mathrm{H}), 3.8(\mathrm{~s}, 3 \mathrm{H}), 3.84(\mathrm{~s}, 3 \mathrm{H}), 5.82(\operatorname{broad~s}, 1 \mathrm{H}), 6.68-67.6(\mathrm{~m}, 3 \mathrm{H}) ;{ }^{13} \mathrm{C} \mathrm{NMR}$ $\left(\mathrm{CDCl}_{3}\right)$ 21.6, 31.7, 34.9, 55.6, 55.7, 61.5, 111.1, 111.7, 120.4, 131.2, 147.3, 148.7, 173.2. MS (EI) $\mathrm{m} / z$ (rel intensity) $263\left(\mathrm{M}^{+}-\mathrm{H}_{2} 0,7\right), 164$ (11), 151 (6), 112 (100), 91 (2), 69 (10), 55 (11). HRMS calcd for $\mathrm{C}_{15} \mathrm{H}_{23} \mathrm{NO}_{4}$ : 218.1627. Found: 218.1621.

The hydroxypentanamide I was submitted to Swern oxidation without purification: DMSO (1.21 mL, $17.1 \mathrm{mmol})$ was added over a solution of oxalyl chloride $(0.75 \mathrm{~mL}, 8.5 \mathrm{mmol})$ in $\operatorname{dry~} \mathrm{CH}_{2} \mathrm{Cl}_{2}(15 \mathrm{~mL})$ at - $60{ }^{\circ} \mathrm{C}$. After stirring for $10 \mathrm{~min}$, a solution of $\mathbf{I}(2 \mathrm{~g}, 7.1 \mathrm{mmol})$ in dry $\mathrm{CH}_{2} \mathrm{Cl}_{2}(80 \mathrm{~mL})$ was added drop wise, and the resulting solution was stirred at $-60{ }^{\circ} \mathrm{C}$ for $1 \mathrm{~h}$. $\mathrm{Et}_{3} \mathrm{~N}(4.9 \mathrm{~mL}, 35.4 \mathrm{mmol})$ was added and, after $15 \mathrm{~min}$, the reaction mixture was allowed to warm up to $\mathrm{rt}$, and stirred at this temperature for $30 \mathrm{~min}$. Water was added $(50 \mathrm{~mL})$, and the organic phase was separated. The resulting aqueous phase was extracted with $\mathrm{CH}_{2} \mathrm{Cl}_{2}(3 \times 50 \mathrm{~mL})$. The combined organic extracts were washed with brine $(3 \times 50 \mathrm{~mL})$, dried $\left(\mathrm{Na}_{2} \mathrm{SO}_{4}\right)$, and concentrated in vacuo to afford $N$-[2-(3,4dimethoxyphenyl)ethyl]-5-oxopentanamide (II) as a colorless oil, that was used without purification : ${ }^{1} \mathrm{H}$ NMR $\left(\mathrm{CDCl}_{3}\right)$ 1.87-1.96 (m, 2H), $2.15(\mathrm{t}, J=7.1,2 \mathrm{H}), 2.73(\mathrm{t}, J=7.1 \mathrm{~Hz}, 2 \mathrm{H}), 3.45-367$ (m, 4H), $3.83(\mathrm{~s}, 3 \mathrm{H}), 3.84(\mathrm{~s}, 3 \mathrm{H}), 5.61($ broad $\mathrm{s}, 1 \mathrm{H}) ; 6.59-6.80(\mathrm{~m}, 3 \mathrm{H}) ; 9.71(\mathrm{~s}, 1 \mathrm{H})$. Oxopentanamide II was 
submitted to cyclization with TFA, according to the procedure described in Procedure A, yielding benzoquinolizidone $\mathbf{2 a}$ ( $1.7 \mathrm{~g}, 92 \%$ two steps), whose data were identical to those reported above.

\section{Synthesis of 9,10-dimethoxy-11b-methyl-1,2,3,6,7,11b-hexahydrobenzo[a]quinolizin-4-one (2b).}

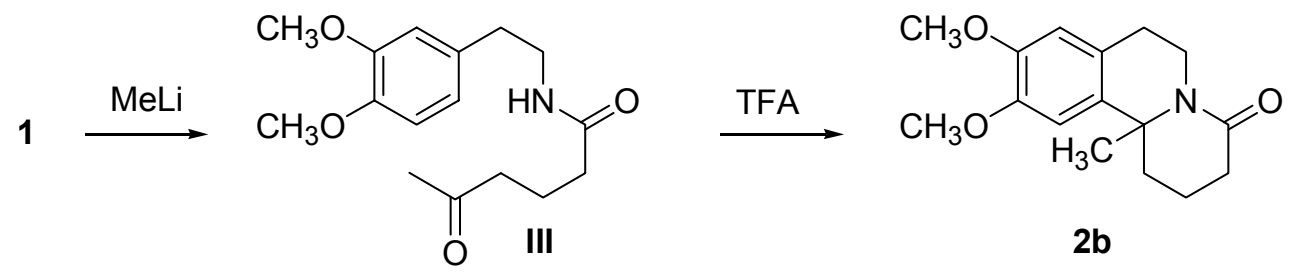

MeLi (4.5 mL of a $1 \mathrm{M}$ solution, $4.5 \mathrm{mmol}$ ) was added drop wise over a solution of glutarimide 1 (418 $\mathrm{mg}, 1.50 \mathrm{mmol})$ in dry $\operatorname{THF}(15 \mathrm{~mL})$ at $-78^{\circ} \mathrm{C}$, and the resulting solution was stirred at this temperature for $6 \mathrm{~h} . \mathrm{H}_{2} \mathrm{O}(10 \mathrm{~mL})$ was added, and the mixture was allowed to reach rt. The organic phase was separated and the aqueous phase was extracted with $\mathrm{Et}_{2} \mathrm{O}(10 \mathrm{~mL})$ and with $\mathrm{CH}_{2} \mathrm{Cl}_{2}(3 \times 10$ $\mathrm{mL})$. The combined organic extracts were washed with brine $(3 \times 10 \mathrm{~mL})$, dried $\left(\mathrm{Na}_{2} \mathrm{SO}_{4}\right)$, and concentrated in vacuo. Flash column chromatography (silica gel, AcOEt) afforded $N$-[2-(3,4dimethoxyphenyl)ethyl]-5-oxohexananamide (III) as a white solid (400 $\mathrm{mg}, 90 \%)$ : $\mathrm{mp}$ (50\% hexane/AcOEt); IR (KBr) 3400, 1730, $1650 \mathrm{~cm}^{-1} ;{ }^{1} \mathrm{H}$ NMR $\left(\mathrm{CDCl}_{3}\right) 1.73$ (q, $\left.J=7.1 \mathrm{~Hz}, 2 \mathrm{H}\right), 1.99$ (s, 3H), $2.04(\mathrm{t}, J=7.1 \mathrm{~Hz}, 2 \mathrm{H}), 2.34(\mathrm{t}, J=7.1 \mathrm{~Hz}, 2 \mathrm{H}), 2.64(\mathrm{t}, J=7.1 \mathrm{~Hz}, 2 \mathrm{H}), 3.28-3.46(\mathrm{~m}, 2 \mathrm{H}), 3.72$, (s, 3H), $3.73(\mathrm{~s}, 3 \mathrm{H}), 6.08-6.12(\mathrm{~m}, 1 \mathrm{H}), 6.59-6.69(\mathrm{~m}, 3 \mathrm{H},) ;{ }^{13} \mathrm{C} \mathrm{NMR}\left(\mathrm{CDCl}_{3}\right)$ 19.5, 29.8, 35.1, 35.2, 40.5, 42.2, 55.7, 111.1, 111.6, 120.4, 131.2, 147.5, 148.7, 172.2, 208.5. MS (EI) $\mathrm{m} / \mathrm{z}$ (rel intensity) 293 $\left(\mathrm{M}^{+}, 2\right), 164$ (100),151 (17), 91 (5), 85 (10), 77 (5). Anal. Calcd for $\mathrm{C}_{16} \mathrm{H}_{23} \mathrm{NO}_{4}: \mathrm{C}, 65.50 ; \mathrm{H}, 7.90$; $\mathrm{N}$, 4.77. Found: C, 65.08; H, 7.75; N, 4.70.

TFA ( $1 \mathrm{~mL}, 12 \mathrm{mmol})$ was added over a solution of oxoamide III (360 mg, $1.2 \mathrm{mmol})$ in $\mathrm{CH}_{2} \mathrm{Cl}_{2}$ (30 $\mathrm{mL}$ ), and the mixture was stirred at $\mathrm{rt}$ for $16 \mathrm{~h}$. The reaction was quenched by addition of saturated $\mathrm{NaHCO}_{3}(10 \mathrm{~mL})$. The organic phase was separated, and the resulting aqueous phase was extracted with $\mathrm{CH}_{2} \mathrm{Cl}_{2}(3 \times 10 \mathrm{~mL})$. The combined organic extracts were washed with brine $(3 \times 10 \mathrm{~mL})$, dried $\left(\mathrm{Na}_{2} \mathrm{SO}_{4}\right)$, and concentrated in vacuo. Flash column chromatography (silica gel, AcOEt) afforded 
benzoquinolizidone $\mathbf{2 b}$ (310 mg, 93\%; overall yield from 1, 84\%): mp (50\% hexane/AcOEt) $92-93{ }^{\circ} \mathrm{C}$ (lit. $\left.{ }^{5} \mathrm{mp} 97-98{ }^{\circ} \mathrm{C}\right)$; IR (KBr) $1610 \mathrm{~cm}^{-1} ;{ }^{1} \mathrm{H}$ NMR $\left(\mathrm{CDCl}_{3}\right) 1.54(\mathrm{~s}, 3 \mathrm{H}), 1.62-1.84(\mathrm{~m}, 3 \mathrm{H}), 2.20-2.34$ $(\mathrm{m}, 2 \mathrm{H}), 2.42-2.59(\mathrm{~m}, 2 \mathrm{H}), 2.74-2.87(\mathrm{~m}, 2 \mathrm{H}), 3.77(\mathrm{~s}, 3 \mathrm{H}), 3.78(\mathrm{~s}, 3 \mathrm{H}), 4.85(\mathrm{dd}, J=13.0,6.8 \mathrm{~Hz}$, $1 \mathrm{H}), 6.50(\mathrm{~s}, 1 \mathrm{H}), 6.56(\mathrm{~s}, 1 \mathrm{H}) ;{ }^{13} \mathrm{C} \mathrm{NMR}\left(\mathrm{CDCl}_{3}\right)$ 16.7, 27.5, 28.6, 31.5, 35.4, 37.2, 55.7, 55.8, 58.4, 108.1, 111.1, 125.0, 134.6, 147.2, 147.3, 168.8; MS (EI) $m / z$ (rel intensity) $275\left(\mathrm{M}^{+}, 7\right), 260$ (100), 244 (5), 216 (5), 190 (4), 131 (2), 91 (3), 77 (4), 55 (10). Anal. Calcd for $\mathrm{C}_{16} \mathrm{H}_{21} \mathrm{NO}_{3}$ : C, 69.79; H, 7.69; N, 5.08. Found: C, 69.45; H, 7.65; N, 4.90 .

\section{Synthesis of 9,10-dimethoxy-1,6,7,11b-tetrahydrobenzo[a]quinolizin-4-one (3a).}<smiles>COc1cc2c(cc1OC)C1CC=CC(=O)N1CCc1ccccc1-2</smiles>

A solution of 2a $(1.04 \mathrm{~g}, 4 \mathrm{mmol})$ in THF $(100 \mathrm{~mL})$ was added drop wise over a solution of LDA [12 mmol, prepared from $i-\mathrm{Pr}_{2} \mathrm{NH}(1.67 \mathrm{~mL}, 12 \mathrm{mmol})$ and $n$-BuLi ( $8 \mathrm{~mL}$ of a $1.5 \mathrm{M}$ solution in hexanes, 12 $\mathrm{mmol})]$ in THF $(20 \mathrm{~mL})$, at $-78^{\circ} \mathrm{C}$. After $40 \mathrm{~min}$, a solution of $\mathrm{PhSeBr}(1,3 \mathrm{~g}, 5.2 \mathrm{mmol})$ in THF (30 $\mathrm{mL}$ ) was added drop wise, and the reaction mixture was stirred at $-78^{\circ} \mathrm{C}$ for $1 \mathrm{~h}$. The reaction was quenched by addition of $1 \mathrm{M} \mathrm{HCl}(40 \mathrm{~mL})$. The organic phase was separated, and the resulting aqueous phase was extracted with $\mathrm{CH}_{2} \mathrm{Cl}_{2}(3 \times 50 \mathrm{~mL})$. The combined organic extracts were washed with brine $(3 \times 50 \mathrm{~mL})$, dried $\left(\mathrm{Na}_{2} \mathrm{SO}_{4}\right)$, and concentrated in vacuo. Flash column chromatography (silica gel, AcOEt) afforded a 2:1 diastereomeric mixture of selenides IV (1.13 g, 70\%): Data of the major diastereomer are given: IR (KBr) $1652 \mathrm{~cm}^{-1}$; ${ }^{1} \mathrm{H}$ NMR $\left(\mathrm{CDCl}_{3}\right)$ 1.60-1.76 (m, 1H), 1.93-2.20 (m, 2H), 2.41-2.59 (m, 1H), 2.64-2.66 (m, 1H), 2.76-2.99 (m, 2H), $3.83(\mathrm{~s}, 3 \mathrm{H}), 3.85(\mathrm{~s}, 3 \mathrm{H}), 4.06(\mathrm{dd}, J=9.9$, $6.7 \mathrm{~Hz}, 1 \mathrm{H}), 4.57(\mathrm{dd}, J=9.9,4.4 \mathrm{~Hz}, 1 \mathrm{H}), 4.79-4.86(\mathrm{~m}, 1 \mathrm{H}), 6.58(\mathrm{~s}, 1 \mathrm{H}), 6.60(\mathrm{~s}, 1 \mathrm{H}), 7.28-7.31$ (m, 3H), 7.67-7.70 (m, 2H); ${ }^{13} \mathrm{C}$ NMR $\left(\mathrm{CDCl}_{3}\right)$ 27.9, 28.3, 30.5, 40.6, 42.1, 55.8, 56.0, 56.6, 107.9, 111.5, 127.3, 128.1, 128.5, 129.0, 135.6, 147.6, 147.8, 168.4. MS (EI) $m / z$ (rel intensity) 259 (45), 244 (9), 191 (100), $176(36), 103(7), 91(9), 77(21)$. 
A solution of the diastereomeric mixture of selenides IV $(1.13 \mathrm{~g}, 2.7 \mathrm{mmol})$ in $\mathrm{CH}_{3} \mathrm{CN} / \mathrm{H}_{2} \mathrm{O}(2: 1,80$ $\mathrm{mL})$ was treated with TFA $(2.1 \mathrm{~mL}, 27 \mathrm{mmol})$ at $\mathrm{rt}$. PIDA $(1.1 \mathrm{~g}, 3.3 \mathrm{mmol})$ was added in portions, and the resulting mixture was stirred for $2 \mathrm{~h}$. The reaction mixture was extracted with hexane $(3 \times 30 \mathrm{~mL})$. The combined organic extracts were washed with saturated $\mathrm{NaHCO}_{3}(3 \times 20 \mathrm{~mL})$, dried $\left(\mathrm{Na}_{2} \mathrm{SO}_{4}\right)$, and concentrated in vacuo. Flash column chromatography (silica gel, 30\% hexane/AcOEt) afforded tetrahydrobenzoquinolizidone 3a (526 mg, 75\%; overall yield from 2a, 56\%): $\mathrm{mp}\left(\mathrm{Et}_{2} \mathrm{O}\right) 128-130{ }^{\circ} \mathrm{C}$; IR ( $\mathrm{KBr}) 1665 \mathrm{~cm}^{-1} ;{ }^{1} \mathrm{H}$ NMR $\left(\mathrm{CDCl}_{3}\right)$ 2.15-2.30 (m, 1H), 2.56-2.88 (m, 4H), $3.80(\mathrm{~s}, 6 \mathrm{H}), 4.63-4.77$ $(\mathrm{m}, 2 \mathrm{H}), 5.99(\mathrm{dd}, J=9.5,2.8 \mathrm{~Hz}, 1 \mathrm{H}), 6.55(\mathrm{~s}, 1 \mathrm{H}), 6.58-6.65(\mathrm{~m}, 1 \mathrm{H}), 6.59(\mathrm{~s}, 1 \mathrm{H}) ;{ }^{13} \mathrm{C} \mathrm{NMR}\left(\mathrm{CDCl}_{3}\right)$ $28.7,33.4,37.7,55.6,55.8,56.7,108.3,111.2,125.2,126.8,127.3,138.8,147.6,147.7,164.5 ;$ MS (EI) $m / z$ (rel intensity) $260\left(\mathrm{M}^{+}+1,15\right), 259\left(\mathrm{M}^{+}, 83\right), 244$ (7), 228 (11), 191 (100), 176 (32), 164 (3), 91 (3). Anal. Calcd for $\mathrm{C}_{15} \mathrm{H}_{17} \mathrm{NO}_{3}$ : C, 69.48; H, 6.61; N, 5.40. Found: C, 69.47; H, 6.77; N, 5.58.

\section{Synthesis of 9,10-dimethoxy-11b-methyl-1,6,7,11b-tetrahydrobenzo[a]quinolizin-4-one (3b).}<smiles>COc1cc2c(cc1OC)C1(C)CC=CC(=O)N1CC2</smiles>

$3 b$

A solution of $\mathbf{2 b}(248 \mathrm{mg}, 0.9 \mathrm{mmol})$ in THF $(10 \mathrm{~mL})$ was added drop wise over a solution of LDA [2.2 mmol, prepared from $i-\mathrm{Pr}_{2} \mathrm{NH}(0.32$ $\mathrm{mL}, 2.2 \mathrm{mmol})$ and $n$-BuLi (3.14 $\mathrm{mL}$ of a $0.7 \mathrm{M}$ solution in hexanes, 2.2 $\mathrm{mmol})]$ in THF $(2 \mathrm{~mL})$, at $-78^{\circ} \mathrm{C}$. After $40 \mathrm{~min}$, a solution of $\mathrm{PhSeBr}$ (300 $\mathrm{mg}, 1.2 \mathrm{mmol})$ in THF $(7 \mathrm{~mL})$ was added drop wise, and the reaction mixture was stirred at -78 ${ }^{\circ} \mathrm{C}$ for $1 \mathrm{~h}$. The reaction was quenched by addition of $1 \mathrm{M} \mathrm{HCl}(40 \mathrm{~mL})$. The organic phase was separated, and the resulting aqueous phase was extracted with $\mathrm{CH}_{2} \mathrm{Cl}_{2}(3 \times 50 \mathrm{~mL})$. The combined organic extracts were washed with brine $(3 \times 50 \mathrm{~mL})$, dried $\left(\mathrm{Na}_{2} \mathrm{SO}_{4}\right)$, and concentrated in vacuo. A solution of the diastereomeric mixture of selenides in $\mathrm{CH}_{2} \mathrm{Cl}_{2}(20 \mathrm{~mL})$ was treated with pyridine $(0.25$ $\mathrm{mL}, 3.1 \mathrm{mmol})$ and $\mathrm{H}_{2} \mathrm{O}_{2}(0.45 \mathrm{~mL}, 6.2 \mathrm{mmol})$ at $0{ }^{\circ} \mathrm{C}$. The resulting mixture was stirred for $1 \mathrm{~h}$ at this temperature, allowed to reach $\mathrm{rt}$, and stirred $16 \mathrm{~h}$. The reaction was quenched by addition of $1 \mathrm{M} \mathrm{HCl}$ $(10 \mathrm{~mL})$. The aqueous phase was extracted with $\mathrm{CH}_{2} \mathrm{Cl}_{2}(3 \times 15 \mathrm{~mL})$. The combined organic extracts were dried $\left(\mathrm{Na}_{2} \mathrm{SO}_{4}\right)$, and concentrated in vacuo. Flash column chromatography (silica gel, 30\% 
hexane/AcOEt) afforded tetrahydrobenzoquinolizidone $\mathbf{3 b}$ as a colorless oil (lit. ${ }^{6} \mathrm{mp}$ (hexane) $115{ }^{\circ} \mathrm{C}$ ) $(151 \mathrm{mg}, 61 \%$ overall yield from $2 \mathrm{~b})$ : IR (neat) $1654 \mathrm{~cm}^{-1} ;{ }^{1} \mathrm{H} \mathrm{NMR}\left(\mathrm{CDCl}_{3}\right) 1.55(\mathrm{~s}, 3 \mathrm{H}), 2.5(\mathrm{dd}, J=$ 17.8, 2.4 Hz, 1H), 2.64-2.79 (m, 2H), 2.81-2.94 (m, 2H), $3.85(\mathrm{~s}, 3 \mathrm{H}), 3.86(\mathrm{~s}, 3 \mathrm{H}), 4.79-4.85(\mathrm{~m}, 1 \mathrm{H})$, $6.06(\mathrm{dd}, J=9.9,3.1 \mathrm{~Hz}, 1 \mathrm{H}), 6.51-6.57(\mathrm{~m}, 1 \mathrm{H}), 6.58(\mathrm{~s}, 1 \mathrm{H}), 6.59(\mathrm{~s}, 1 \mathrm{H}) ;{ }^{13} \mathrm{C} \mathrm{NMR}\left(\mathrm{CDCl}_{3}\right) 24.8$, $29.1,34.1,39.1,55.8,56.1,57.5,108.3,111.2,124.9,125.9,133.7,137.3,147.6,147.8,163.4$; MS (EI) $m / z$ (rel intensity) $273\left(\mathrm{M}^{+}, 6\right), 259$ (18), 258 (100), 242(6), 214 (4), 205 (3). Anal. Calcd for $\mathrm{C}_{16} \mathrm{H}_{19} \mathrm{NO}_{3}: \mathrm{C}, 70.31 ; \mathrm{H}, 7.06 ; \mathrm{N}, 5.12$. Found: $\mathrm{C}, 69.95 ; \mathrm{H}, 6.86 ; \mathrm{N}, 4.82$.

\section{References}

(1) Still, W. C.; Kann, H.; Miltra, A. J. J. Org. Chem. 1978, 43, 2923-2925.

(2) Perrin, D. D.; Armarego, W. L. F. Purification of Laboratory Chemicals; Pergamon Press: Oxford, 1997.

(3) Collado, M. I.; Manteca, I.; Sotomayor, N.; Villa, M. J.; Lete, E. J. Org. Chem. 1997, 62, 20802092.

(4) Orito, K.; Matsuzaki, T.; Sugiome, H.; Rodrigo, R. Heterocycles 1988, 27, 2403-2412.

(5) Padwa, A.; Waterson, A. C. J. Org. Chem. 2000, 65, 235-244.

(6) Kametani, T.; Terasawa, H.; Ihara, M. J. Chem. Soc. Perkin Trans. 1 1976, 2547-2550. 